Case Report

\title{
Delayed diagnosis of a molar pregnancy in a perimenopausal woman: a case report
}

\section{Ranasinghe RASW ${ }^{1}$, Lankeshwara $\mathbf{D}^{1}$, Lekamge NR $^{1}$, Priyadarshini KHC $^{1}$}

\section{${ }^{1}$ District General Hospital, Monaragala, Sri Lanka}

\section{Introduction}

Gestational Trophoblastic Disease (GTD) is an abnormal proliferation of trophoblastic tissue during pregnancy. It is collectively a spectrum of diseases encompassing complete or partial hydatidiform mole to choriocarcinoma, and may be associated with preeclampsia and/or hyperthyroidism ${ }^{1}$. The incidence of GTD is 1 in 700 pregnancies which is comparatively high in Asiatic women ${ }^{2}$. Hydatidiform mole is the commonest type and usually occurs in extremes of reproductive age.

\section{Case presentation}

A 57-year old perimenopausal Sinhalese woman was admitted to District General Hospital Monaragala with vaginal bleeding for one month. She was a diagnosed hypertensive patient with poor compliance. She lived with her daughter and denied any sexual activity in the recent past. On examination she was pale and had bilateral ankle oedema with a blood pressure of $180 / 90$ mmHG. The abdomen was soft and uterus was just palpable. On speculum examination, cervix was healthy with bleeding through the cervical OS. Her urine hCG was negative on admission. Initially it was planned to proceed to endometrial sampling to investigate her for post menopausal bleeding. However it was postponed due to poor control of blood pressure despite being on multiple antihypertensives.

A week after admission, she developed headache and vomiting with blood pressure of 200/110 $\mathrm{mmHg}$. She was also found to have significant proteinuria. Haemoglobin was $8.8 \mathrm{~g} / \mathrm{dl}$ and white cell count was $5,600 / \mathrm{mm} 3$. Platelet count on admission was $159,000 / \mu \mathrm{l}$ but dropped to $69,000 /$ $\mu \mathrm{l}$ after seven days. Coagulatory profile and renal functions were normal. Liver functions were normal except for mild hypoalbuminemia of 2.89 $\mathrm{g} / \mathrm{dL}$. Echocardiogram did not show changes of long standing hypertension. Serum $\beta$-hCG was $410,208 \mathrm{mIU} / \mathrm{ml}$. Her urine hCG became positive when it was diluted. Ultrasonographic evidence showed $3.2 \mathrm{~cm}$ thickened uterine wall and large heterogenous lesion with multiple cystic areas inside the cavity without extra uterine invasion (Fig. 1).

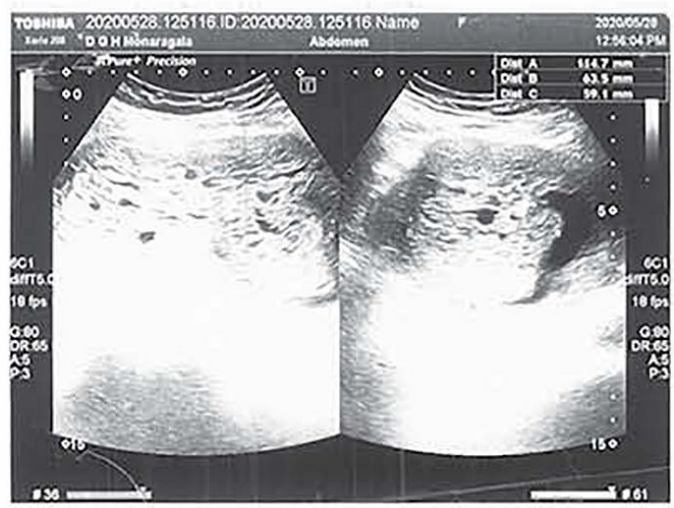

Fig. 1 Sonographic image of uterus showing a heterogenous lesion with multiple cystic areas

She was started on Magnesium sulphate. A laparotomy with total abdominal hysterectomy and bilateral salpingo-oophorectomy with bilateral internal iliac artery ligation was done. Uterus was filled with vesicles (Fig.2).

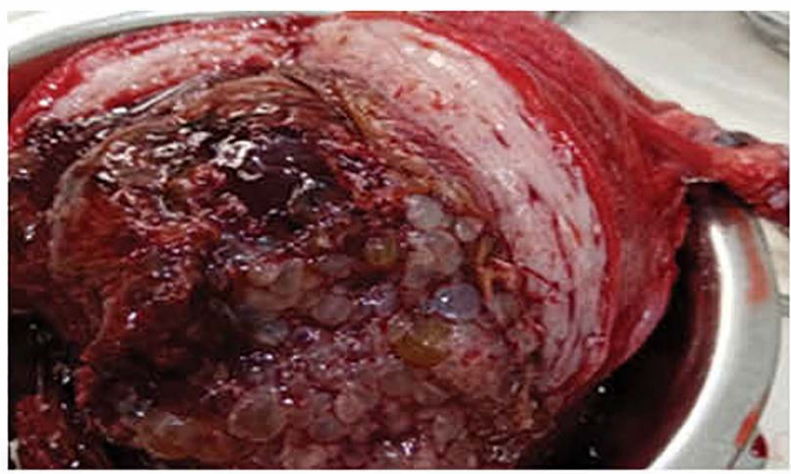

Fig. 2 Macroscopic appearance of uterus 
One unit of blood and ten units of platelets were transfused in the immediate post-operative period. Microscopic examination demonstrated multiple hydrophic villi with excessive trophoblastic proliferation without myometrial invasion suggestive of benign complete hydatidiform mole (Fig.3).

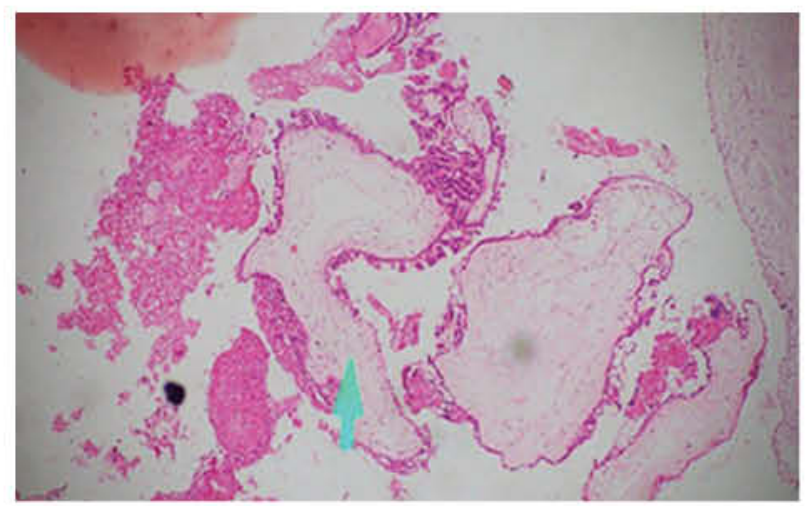

Fig. 3 Trophoblastic proliferation

Follow-up showed a rapid fall in serum $\beta$-hCG level to $853 \mathrm{mIU} / \mathrm{ml}$ following three weeks of surgery. $\mathrm{X}$ ray chest and Computed tomography of brain were normal.

\section{$\underline{\text { Discussion }}$}

This patient had GTD with severe preeclampsia and disseminated intravascular coagulation. There have been several reported cases of GTD with complications in perimenopausal women ${ }^{3,4}$. This case emphasizes the importance of considering GTD as a cause for abnormal uterine bleeding in women of advanced age.

Confirmation of pregnancy in women of reproductive age is often based on urine hCG in low resourced settings. It is a qualitative test that detects $\beta$ hCG in urine. Unfortunately there may be false negative results when $\beta$ hCG values are very high as in GTD. This phenomenon is known as 'high dose hook effect'1,5. Therefore, urinary hCG can be misleading and may lead to inappropriate treatment and catastrophic outcomes.

\section{Conclusion}

GTD should be included in differential diagnosis of abnormal uterine bleeding in peri-menopausal age. Prompt diagnosis and appropriate management are necessary as the diagnosis of GTD is often not considered in this age group. It underscores the importance of knowing the quantitative serum beta hCG due to limitations of urine hCG strips.

\section{Keywords:}

Beta-human Chorionic Gonadotropin, Gestational Trophoblastic Disease, Post menopausal woman

\section{$\underline{\text { Acknowledgements }}$}

We express our sincere gratitude to the members of obstetrics and gynaecology unit, District General Hospital, Monaragala.

\section{Competing interests}

The authors declare that they have no competing interests.

\section{$\underline{\text { References }}$}

1) Christopher L, Hunter MD, Ladde J. Molar Pregnancy with False Negative $\beta$-hCG Urine in the Emergency Department. Western Journal of Emergency Medicine 2011; 12(2):213-215

2) Bracken MB, Brinton LA, Hayashi K. Epidemiology of hydatidiform mole and choriocarcinoma. Epidemiologic Reviews 1984; 6:52-75

3) Fouchardière $\mathrm{A}$, Cassignol $\mathrm{A}$, Benkiran $\mathrm{A}$, et al. Invasive hydatiform mole in a postmenopausal woman. Ann Pathology 2003; 23(5):443-446

4) Mehrotra S, Singh U, Chauhan S. Molar pregnancy in postmenopausal women: a rare phenomenon. BMJ Case Report 2012.

5) Nigam A, Kumari A, Gupta N. Negative Pregnancy test in molar pregnancy; Is it possible. Case report. BMJ Case Rep 2014 